\title{
Stimulatory Effects of Sugarcane Molasses on Fumigaclavine C Biosynthesis by Aspergillus fumigatus CY018 via Biofilm Enhancement ${ }^{\text {[S }}$
}

\author{
Jun Tao, Fa-Liang An, Zheng-Hua Pan, and Yan-Hua Lu* \\ State Key Laboratory of Bioreactor Engineering, East China University of Science and Technology, Shanghai 200237, P.R. China
}

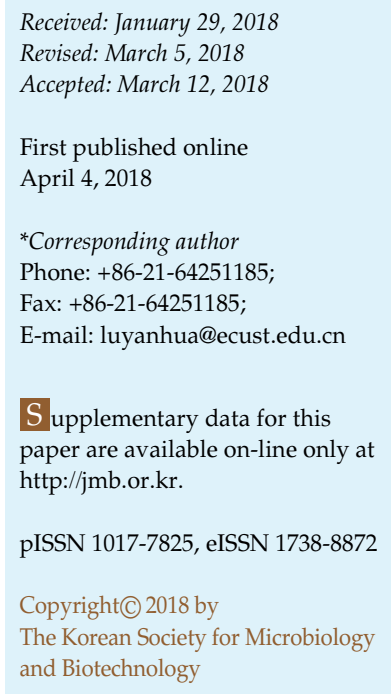

\begin{abstract}
Biofilms are of vital significance in bioconversion and biotechnological processes. In this work, sugarcane molasses was used to enhance biofilms for the improvement of the production of fumigaclavine C (FC), a conidiation-associated ergot alkaloid with strong anti-inflammatory activities. Biofilm formation was more greatly induced by the addition of molasses than the addition of other reported biofilm inducers. With the optimal molasses concentration (400 g/l), the biofilm biomass was 6-fold higher than that with sucrose, and FC and conidia production was increased by 5.8- and 3.1-fold, respectively. Moreover, the global secondary metabolism regulatory gene $l a e A$, FC biosynthetic gene $f g a O x 3$, and asexual central regulatory genes brlA and wet $A$ were upregulated in molasses-based biofilms, suggesting the upregulation of both asexual development and FC biosynthesis. This study provides novel insight into the stimulatory effects of molasses on biofilm formation and supports the widespread application of molasses as an inexpensive raw material and effective inducer for biofilm production.
\end{abstract}

Keywords: Fumigaclavine C, sugarcane molasses, biofilm, Aspergillus fumigatus CY018

\section{Introduction}

Fumigaclavine C (FC) is a conidiation-associated ergot alkaloid isolated from the filamentous endophytic fungus Aspergillus fumigatus CY018 [1]. It displays strong antiinflammatory activity $[2,3]$, as well as antitumor and antiatherogenic effects $[4,5]$, indicating the potential of this clavine alkaloid for widespread application.

FC (shown in Fig. S1), composed of a tetracyclic ergoline ring, is the end product of the ergot alkaloid pathway in $A$. fumigatus [6]. The biosynthesis of FC is initiated by the prenylation of L-tryptophan in the presence of dimethylallyl diphosphate [7]. Through several biosynthetic steps, including methylation, acetylation, cyclization, and prenylation, the major intermediates are ultimately converted to FC [8]. FgaOx1 and FgaOx3 are involved in the cyclization of 4-dimethylallyl-L-abrine and chanoclavine-1 aldehyde, respectively, to form tricyclic and tetracyclic ergoline scaffolds; these enzymes are coordinated by the global secondary metabolic regulator LaeA [9]. Additionally, the expression of BrlA, the central activator of A. fumigatus conidiation, is indispensable for FC biosynthesis [10], indicating an interesting correlation between asexual development and FC biosynthesis. Moreover, several conidiation-inducing methods, such as ultrasonic stimulation and two-stage procedures, can effectively result in FC production [1, 11]. However, further investigation is required to examine the stimulatory effects of conidiation on genes involved in FC biosynthesis.

A. fumigatus conidiation is observed during biofilm maturation, which, along with the planktonic state, is a natural stage of fungal growth [12]. Biofilm growth is considered a developmental shift towards preservation, wherein structured polymicrobial biofilms form on natural or inanimate surfaces [13]. Fungal biofilms are multicellular communities that possess vastly distinct phenotypes compared with their planktonic counterparts [14]. Moreover, biofilms are of vital significance in bioconversion and biotechnological processes. In one of the earliest publications on the topic, Aspergillus niger biofilm was adhered to a vertical disc rotating fermenter, and a 4 -fold increase in the production of citric acid was observed [15-17]. It was also reported that $A$. niger biofilm attached to the surface of polyurethane foam in a rotating biological contactor attained 
3-fold higher citric acid production than in a stirred-tank fermenter [18]. Biofilms perform better at producing a wide range of bioproducts, such as kojic acid by Aspergillus oryzae [19], fructooligosaccharides by Aspergillus phoenicis [20], red pigment by Penicillium novae-zelandiae [21], and cellulase by $A$. niger [22]. Gliotoxin, one of the major secondary metabolites produced by A.fumigatus, increases with biofilm formation, and several genes involved in secondary metabolic biosynthesis are significantly upregulated in the mature phase [23]. Nevertheless, whether the biosynthesis of FC, another secondary metabolite produced by $A$. fumigatus, is related to biofilm formation remains unknown.

Previous studies have evaluated sugarcane molasses, a low-cost waste feedstock, as an efficient supplemental ingredient in two-stage culture medium [24]. However, the mechanism underlying the promotion of conidiation and FC biosynthesis in A. fumigatus by molasses remains unknown. In this work, the distributions of conidia and FC were examined, and found to be concentrated in the biofilm instead of the planktonic state. Several additives reported to enhance biofilm formation in other studies were tested with A. fumigatus, and molasses was observed to be an excellent additive that contains various carbon sources. The concentration of molasses as a sole carbon source was optimized and compared with sucrose. Biofilm morphologies were observed via scanning electron microscopy (SEM). Furthermore, the expression profiles of genes involved in FC biosynthesis (laeA, fgaOx1, and $\left.f_{g} a O x 3\right)$ and asexual development (brlA, wet $A$, and $\left.a b a A\right)$ were analyzed to elucidate transcriptional changes in biofilm cells in molasses-based medium. This study suggests the widespread application of molasses as an inexpensive raw material and effective inducer for biofilm production, and provides useful information for further investigation on FC biosynthetic regulation.

\section{Materials and Methods}

\section{Strain and Culture Conditions}

A. fumigatus CY018, provided by Nanjing University, was maintained on potato-dextrose-agar (PDA) slants and cultured at $28^{\circ} \mathrm{C}$ for 7 days. Afterwards, 2 agar blocks $(10 \mathrm{~mm} \times 10 \mathrm{~mm})$ were transferred to a $500-\mathrm{ml}$ shake flask containing $200 \mathrm{ml}$ of potatodextrose broth. After shaking at $150 \mathrm{rpm}$ and $28^{\circ} \mathrm{C}$ for $72 \mathrm{~h}, 7 \mathrm{ml}$ of the seed broth was inoculated in a 250-ml Erlenmeyer flask containing $43 \mathrm{ml}$ of fermentation medium. Each liter of the cultivation medium contained $1 \mathrm{~g}$ of $\mathrm{NaNO}_{3}, 1.5 \mathrm{~g}$ of $\mathrm{KH}_{2} \mathrm{PO}_{4}$, $0.7 \mathrm{~g}$ of $\mathrm{Mg}_{2} \mathrm{SO}_{4}, 0.02 \mathrm{~g}$ of $\mathrm{FeSO}_{4} \cdot 7 \mathrm{H}_{2} \mathrm{O}, 3 \mathrm{~g}$ of sodium glutamate, $1.5 \mathrm{~g}$ of sodium tartrate, and either $60 \mathrm{~g}$ of sucrose or different concentrations of molasses as a carbon source. The final $\mathrm{pH}$ of the medium was adjusted to 5.2 with $1 \mathrm{M} \mathrm{HCl}$. FC was produced in a two-stage process. First, the culture was incubated at $28^{\circ} \mathrm{C}$ and $130 \mathrm{rpm}$ for $72 \mathrm{~h}$, and then the culture was moved to a $9-\mathrm{cm}-$ diameter plate for static biofilm formation.

\section{Sampling and Determination of Biomass, Conidia, and Fumigaclavine C Production}

Three flasks or plates were sampled in each experimental replicate. Biomass production was examined by determining the dry cell weight (DCW) during different fungal phases. For planktonic samples, the broth was filtered through a preweighed filter paper under suction and dried in an oven at $60^{\circ} \mathrm{C}$ to a constant weight; for biofilm samples, the biofilm was washed three times with distilled water and dried as described above [22]. The number of conidia was determined with a hemocytometer.

The broth and the biofilm were separated, extracted 3 times with $50 \mathrm{ml}$ of ethyl acetate, and evaporated using a vacuum rotary evaporator at $45^{\circ} \mathrm{C}$. The crude extract was dissolved with methanol and filtered by a $0.22-\mu \mathrm{m}$ polyvinylidene difluoride syringe filter for high-performance liquid chromatography analysis. An Agilent ZORBAX Eclipse SB-C18 column $(4.6 \mathrm{~mm} \times 250 \mathrm{~mm}, 5 \mu \mathrm{m})$ was used for quantitative analysis of FC. Analytes $(20 \mu \mathrm{l})$ were injected and eluted in the mobile phase in solvent $\mathrm{A}$ ( $35 \%$ acetonitrile) and solvent $\mathrm{B}\left(65 \% \mathrm{H}_{2} \mathrm{O}, 1 \%\right.$ trifluoroacetic acid) at the flow rate of $1 \mathrm{ml} / \mathrm{min}$ for $25 \mathrm{~min}$ in an Agilent 1290 Infinity LC system. The UV absorption was measured at $227 \mathrm{~nm}$ and the operating temperature was set at $25^{\circ} \mathrm{C}$. FC concentrations were determined using a standard curve prepared with FC powder provided by Prof. RX Tan, Nanjing University.

\section{Biofilm Quantification}

The biofilm biomass was estimated according to a modified Crystal Violet staining method [25]. In brief, A. fumigatus biofilms were formed on commercially available 96-well microtiter plates after 3 days of shaking in flasks. A standardized cell suspension $(200 \mu \mathrm{l})$ was statically incubated for 8 days. After incubation, the culture medium was removed, and the biofilm was washed at least 3 times with phosphate-buffered saline (PBS), stained with $200 \mu \mathrm{l}$ of $0.1 \%$ Crystal Violet for $5 \mathrm{~min}$, and washed with deionized water to remove excess dye. The biofilms were destained with $200 \mu \mathrm{l}$ of $95 \%$ ethanol, $150 \mu \mathrm{l}$ of the solution was transferred into a clean 96-well microtiter plate, and the absorbance was measured at $570 \mathrm{~nm}$. The absorbance values were proportional to the biofilm biomass, which comprises the hyphae and extracellular polymeric substances.

\section{Scanning Electron Microscopy}

SEM was used to observe the morphology of A. fumigatus using sucrose or molasses as a carbon source. Cells in different growth phases were fixed in $2.5 \%(\mathrm{v} / \mathrm{v})$ glutaraldehyde at $4^{\circ} \mathrm{C}$ for $12 \mathrm{~h}$. The cells were washed twice in PBS and dehydrated with an ethanol concentration gradient. Finally, imaging was performed on a scanning electron microscope (S-3400; Hitachi, Japan). 
Measurement of Gene Expression by Real-Time Quantitative Polymerase Chain Reaction

Total RNA was extracted from $100 \mathrm{mg}$ of fresh cells via the RNAiso Plus kit (Takara Bio Inc., Japan), and cDNA was prepared with the PrimeScript RT reagent kit according to the manufacturer's protocols.

The transcriptional levels of $b r l A, a b a A$, wetA, fgaOx1, fgaOx3, and lae $A$ were analyzed using a CFX96 Real-Time PCR Detection System (Bio-Rad, USA). Each $25 \mu$ real-time quantitative polymerase chain reaction (qPCR) contained $2 \mu \mathrm{l}$ of $\mathrm{CDNA}$, and was performed using the SYBR Premix Ex Taq II kit (Takara Bio Inc., Japan). After $30 \mathrm{sec}$ of denaturation at $95^{\circ} \mathrm{C}$, the cDNA was amplified with 40 cycles of denaturation at $95^{\circ} \mathrm{C}$ for $5 \mathrm{sec}$, annealing at $55^{\circ} \mathrm{C}$ for $30 \mathrm{sec}$, and extension at $72^{\circ} \mathrm{C}$ for $60 \mathrm{sec}$. Identical thermal cycling conditions were utilized for all targets, and the transcriptional levels were normalized to A. fumigatus $\beta$-actin. Each reference sample was set as 1.0, and the results of others were expressed as fold-increase in mRNA level over reference samples. Primers for target genes are presented in Table 1.

\section{Statistical Analysis}

All data represent the mean \pm standard deviation of three independent experiments. One-way analysis of variance was used to determine the significance of differences between cultures under different conditions. A value of $p<0.05$ was considered significant.

\section{Results and Discussion}

\section{Distribution of Conidia and Fumigaclavine C in Molasses- Containing Culture}

In our previous work, aerial mycelia (i.e., biofilm) were

Table 1. Primers used for the qPCR of Aspergillus fumigatus gene expression.

\begin{tabular}{|c|c|}
\hline Gene & Primer sequence \\
\hline \multirow[t]{2}{*}{$\beta$-actin } & F: 5'-AGACCTTCAACGCTCCCGCCT-3' \\
\hline & R: 5'-GGCGTGGGGGAGAGCGAAAC-3' \\
\hline \multirow[t]{2}{*}{ brlA } & F: 5'-ACACCAAGACCCACAGCAAA-3' \\
\hline & R: 5'-AGTAAGTGGCCCCCGATAGT-3' \\
\hline \multirow[t]{2}{*}{ wetA } & F: 5'-AAGCCCGTCAAAGGGCCAAA-3' \\
\hline & R: 5' -AACTCCCACTCGGAGCGACT-3' \\
\hline \multirow[t]{2}{*}{$a b a A$} & F: 5' -CAGGGCGACAAAGCCTTCCA-3' \\
\hline & R: 5'-ACATTCTCCAGCGGCATGGG-3' \\
\hline \multirow[t]{2}{*}{ laeA } & F: 5'- GTTGGCACGCCAATGGTTCC-3' \\
\hline & R: 5'- CGGCAGCTCGAAAGAAGGGA-3' \\
\hline \multirow[t]{2}{*}{$f g a O x 1$} & F: 5'- TTTCCTTGACACCCATCCCC-3' \\
\hline & R: 5'- TCTTCAGTGCGACCAACCTC-3' \\
\hline \multirow[t]{2}{*}{$f g a O x 3$} & F: 5'- TGAGGTGTTTGTGCGTGTCT-3' \\
\hline & R: 5'- GCCACATTGGTGTAAGTCGC-3' \\
\hline
\end{tabular}

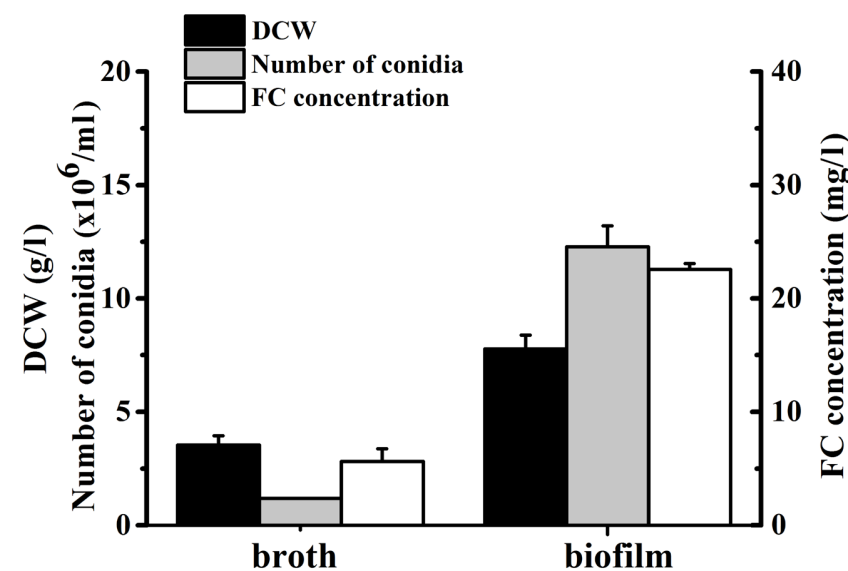

Fig. 1. Distribution of dry cell weight (DCW), number of conidia, and fumigaclavine C (FC) production in the broth versus biofilm from $60 \mathrm{~g} / 1$ sucrose on day 12 of fermentation.

observed at the gas-liquid surface, which stimulated conidiation and facilitated oxygen transport during the static culture stage $[1,24]$. Thus, the two phases of growing cells, the planktonic cells in the culture broth and the biofilm on the static surface, coexist during the two-stage process. However, the precise contributions of $A$. fumigatus biofilms and planktonic cells to conidiation and FC production in the two-stage process remain unclear.

The biofilm and culture broth were separated and analyzed with sucrose-based medium. As shown in Fig. 1, the majority of conidia and FC were retained in the biofilm, with 10.5-fold more conidia and 4.0-fold greater FC production in the biofilm, which had a 2.2-fold higher DCW compared with the cells in the broth. Metabolites produced by A. fumigatus in the broth and biofilm were compared (Fig. S2), to further demonstrate the differences between them. The results demonstrate that by-product titers were higher in the broth, whereas the FC concentration was obviously greater in the biofilm. This indicates that FC is the main metabolite produced by the biofilm, and suggests a potential role for biofilm in conidia production and FC biosynthesis. Biofilm had a similar stimulatory impact on the production of gliotoxin, another conidiation-associated metabolite produced by A. fumigatus [23].

\section{Effects of Different Inducers on Biofilm Formation and Fumigaclavine C Production in A. fumigatus}

To attain higher FC production, different additives were tested for the ability to enhance biofilm formation. Different concentrations of additives previously reported to stimulate biofilm formation were evaluated in sucrose-based medium, 


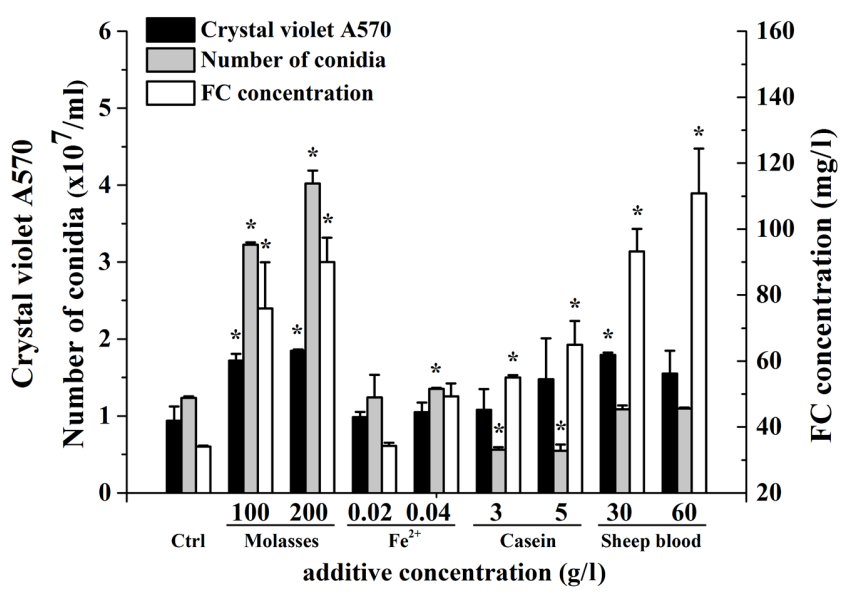

Fig. 2. Effect of different additives on the formation of biofilm (Crystal Violet A570), fumigaclavine C (FC) production, and number of conidia.

Ctrl: $0.94 \pm 0.17$ for Crystal violet A590, $1.23 \pm 0.02 \times 10^{7}$ conidia $/ \mathrm{ml}$ for Number of conidia, and $34.03 \pm 0.32 \mathrm{mg} / 1$ for FC concentration.

including molasses [26], $\mathrm{Fe}^{2+}$ [27], casein [28], and sheep blood [29]. As shown in Fig. 2, the addition of molasses (100 and $200 \mathrm{~g} / \mathrm{l})$ and sheep blood (30 g/l) significantly enhanced biofilm formation after 12 days of fermentation, with 1.83-, 1.97-, and 1.91-fold increases compared with the control, whereas $\mathrm{Fe}^{2+}$ and casein addition slightly increased biofilm growth. At the same time, the production of FC was also markedly improved with the addition of molasses and sheep blood, reaching $89.98 \mathrm{mg} / 1$ and $110.80 \mathrm{mg} / \mathrm{l}$, respectively, compared with $34 \mathrm{mg} / 1$ in the control. Regarding asexual sporulation, molasses markedly increased the conidia number, whereas sheep blood exerted no significant effect. This might be due to the enrichment of nitrogen sources in sheep blood, which inhibit sporulation [30]. The results suggest that both molasses and sheep blood are excellent inducers for biofilm formation and FC production. However, molasses is less expensive and easier to sterilize, and can also serve as a carbon source during fermentation [31]. Therefore, molasses was selected for further study, and biofilms were subjected to further analyses to illuminate the stimulatory effects of molasses on the conidiation and FC production of A. fumigatus.

Enhancement of Biofilm Growth and Fumigaclavine C Production through the Optimization of Molasses Concentration

The biosynthesis of ergot alkaloids requires significant amounts of carbon, primarily sourced from sucrose or glucose [32], which makes it economically unfavorable for

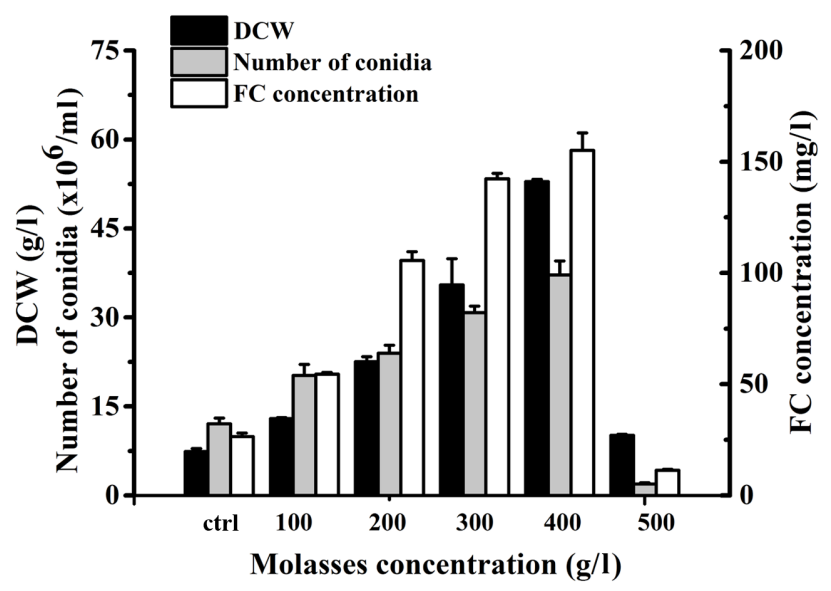

Fig. 3. Effect of molasses concentration on dry cell weight (DCW), number of conidia, and fumigaclavine C (FC) production in the biofilm.

Ctrl: $0.94 \pm 0.17$ for Crystal violet A590, $1.23 \pm 0.02 \times 10^{7}$ conidia $/ \mathrm{ml}$ for Number of conidia, and $34.03 \pm 0.32 \mathrm{mg} / 1$ for FC concentration.

industrial FC production. On the contrary, molasses is a waste product of the sugar industry, and shows promise as a supplementary ingredient for A. fumigatus fermentation. Moreover, molasses, with a similar amount of carbon (i.e., residue sugar) as sucrose, performs better in FC production (Table S1). Furthermore, $60 \mathrm{~g} / 1$ of sucrose was dispensable in medium containing molasses (data not shown); therefore, molasses was utilized as a sole carbon source in the subsequent experiments, which might further decrease fermentation costs. The molasses concentration was optimized to promote biofilm growth. As shown in Fig. 3, A. fumigatus biofilm growth was increased in molassesbased medium harvested on day 12. The DCW of the biofilm increased in a dose-dependent manner, and peaked at $52.9 \mathrm{~g} / 1$ with $400 \mathrm{~g} / 1$ molasses. Conidiation and FC production also increased with increasing molasses concentration. Clearly, $400 \mathrm{~g} / 1$ molasses was optimal for cell growth, conidiation, and FC production, with $37.1 \times 10^{6}$ conidia/ml and $155.1 \mathrm{mg} / 1$ of FC produced. However, this effect was not observed at $500 \mathrm{~g} / 1$ molasses, when the growth of $A$. fumigatus was severely diminished, possibly because of unfavorable osmotic pressure. A. fumigatus macroand microstructures grown in sucrose- and molasses-based media were observed by SEM. Compared with the control in Fig. 4A, the morphology of A. fumigatus grown in $400 \mathrm{~g} / 1$ molasses-based medium (Fig. 4B) formed a much curlier biofilm, whereas $500 \mathrm{~g} / 1$ molasses failed to form a complete biofilm (Fig. 4C). In addition, the biofilm cultivated in molasses-based medium produced markedly more conidia 
A

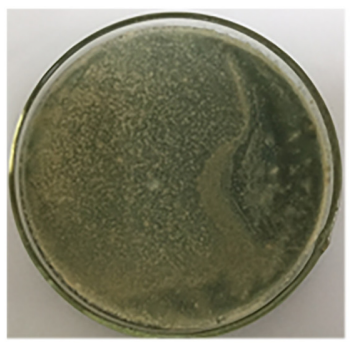

D

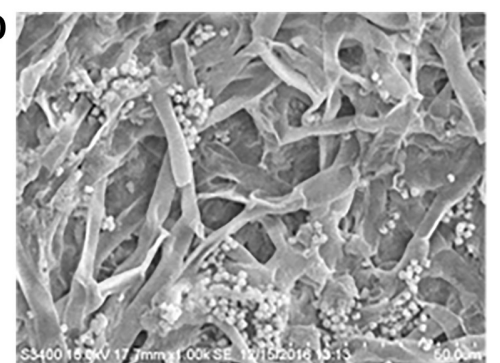

B

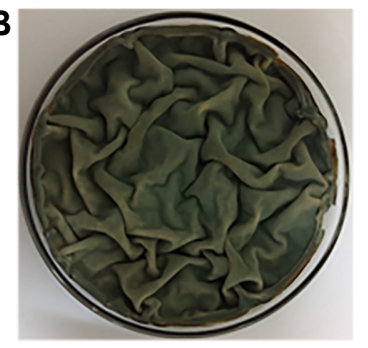

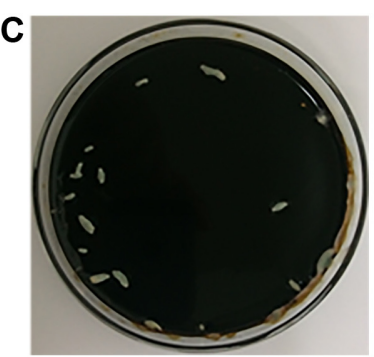

E

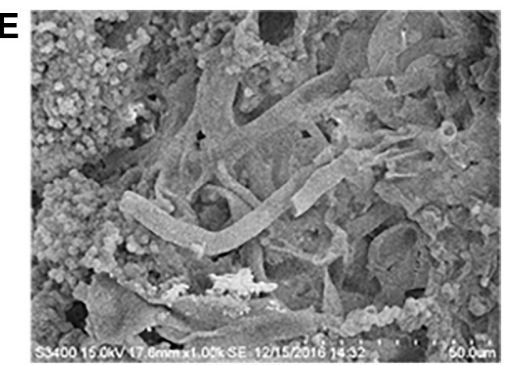

Fig. 4. Macro- (A-C) and micro-structures (D and E) of A. fumigatus grown under $60 \mathrm{~g} / 1$ sucrose-based (A and D) and $400 \mathrm{~g} / \mathrm{l}$ molasses-based (B and E) media after $144 \mathrm{~h}$ of fermentation.

Note that, the macro-structure of A. fumigatus grown in 500 (g/l) molasses (C) was also observed.

with more extracellular matrix (Figs. 4D, 4E), implying the promotion of asexual development with enhanced biofilm formation.

\section{Effects of Molasses as a Sole Carbon Source on Biofilm Growth, Conidiation, and Fumigaclavine C Production}

To study the kinetic effects of molasses on the fermentation process of $A$. fumigatus, biofilm growth, the number of conidia, and FC production were compared in $60 \mathrm{~g} / 1$ sucrose- and $400 \mathrm{~g} / 1$ molasses-based media. As shown in Fig. 5, compared with sucrose, molasses had a positive impact on biofilm DCW throughout the fermentation process. The biofilm DCW of sucrose- and molasses-based media both reached their maximal levels (7.38 and $52.90 \mathrm{~g} / \mathrm{l}$, respectively) after $288 \mathrm{~h}$ of cultivation. Meanwhile, the amount of conidia peaked at $240 \mathrm{~h}\left(12.1 \times 10^{6}\right.$ and $37.1 \times 10^{6}$ conidia/ml, respectively), followed by slight decreases afterwards, which might be due to the germination of a small portion of the conidia. FC biosynthesis began after $96 \mathrm{~h}$ of cultivation, and the highest FC production values of sucrose- and molasses-based media (26.49 and $155.10 \mathrm{mg} / \mathrm{l}$, respectively) were obtained after $288 \mathrm{~h}$. The kinetic data demonstrated a strong facilitating role for molasses in the biofilm growth stage (static stage) of $A$. fumigatus.

\section{A. fumigatus Asexual Development Gene Expression in Molasses-Based Medium}

To better understand the mechanism underlying the stimulatory role of molasses, the transcriptional profiles of A. fumigatus biofilms grown in sucrose- and molassesbased media were examined. Conidiation is the major reproductive mode of aspergilli, and is mainly controlled by three central regulators, BrlA, AbaA, and WetA [33]. The expression of $b r l A$, wet $A$, and $a b a A$ was assayed at the transcriptional level in A. fumigatus biofilms (Fig. 6). In molasses-based medium, brlA expression was significantly upregulated on the first day of static culture, and was 11.24-fold higher than in sucrose-based medium. The expression of wet $A$ and $a b a A$ was downregulated, possibly due to unfavorable components present in the raw molasses [1]. However, these inhibitory effects were reduced by the third day of static culture, possibly as a result of earlier BrlA expression. Molasses promoted the expression of $\mathrm{brlA}$ and wet $A$ for 8 and 6 days, respectively, but for less than 2 days for $a b a A$, suggesting that $b r l A$ and wet $A$ are two major genes affected by molasses. This may be due to wet $A$ feedback inhibition during asexual development signaling [33], which meanwhile verified the upregulation of $b r l A$ and $w e t A$ in the asexual development of A.fumigatus. The results suggest that molasses significantly increases the expression of asexual development genes, especially brlA and wetA, during A. fumigatus biofilm growth.

\section{Fumigaclavine C Biosynthesis Gene Expression in Molasses-Based Medium}

To further investigate the mechanism underlying the 

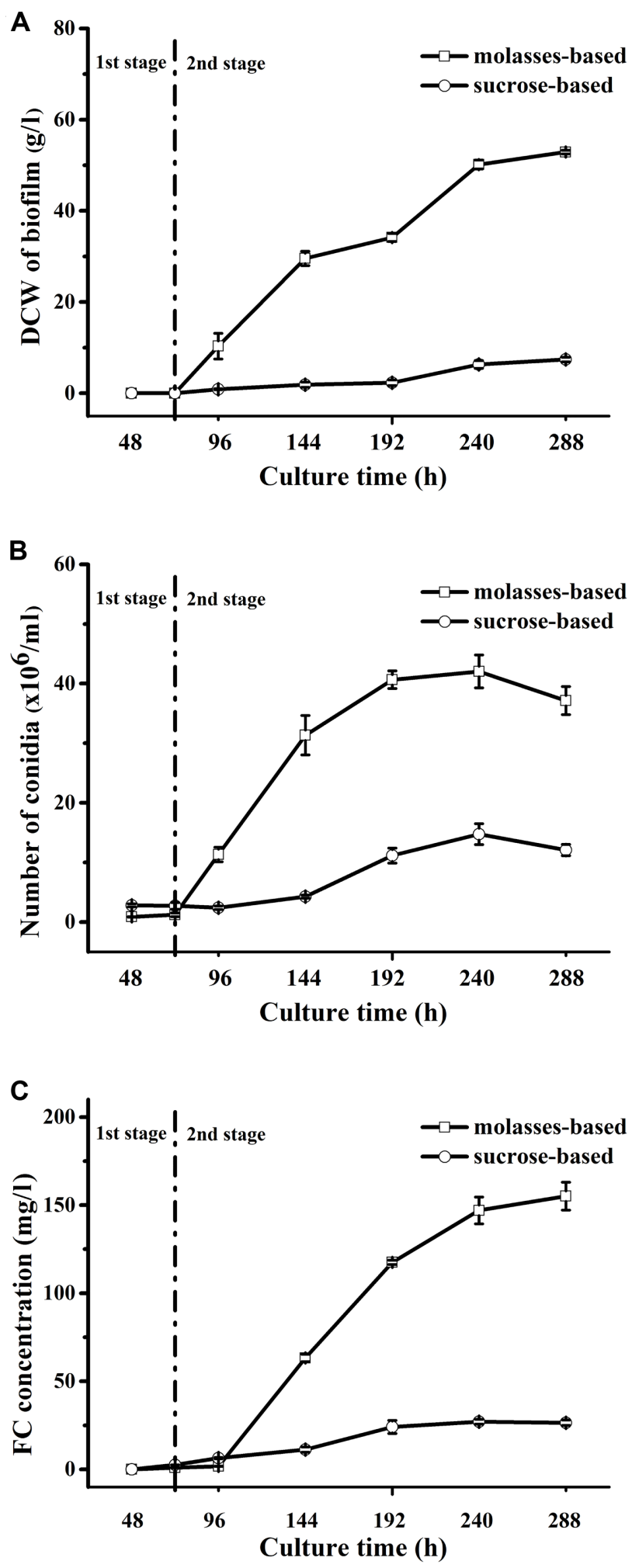

Fig. 5. Time course of dry cell weight (DCW) of biofilm (A), number of conidia (B), and fumigaclavine C (FC) production (C) in sucrose-based and molasses-based media.
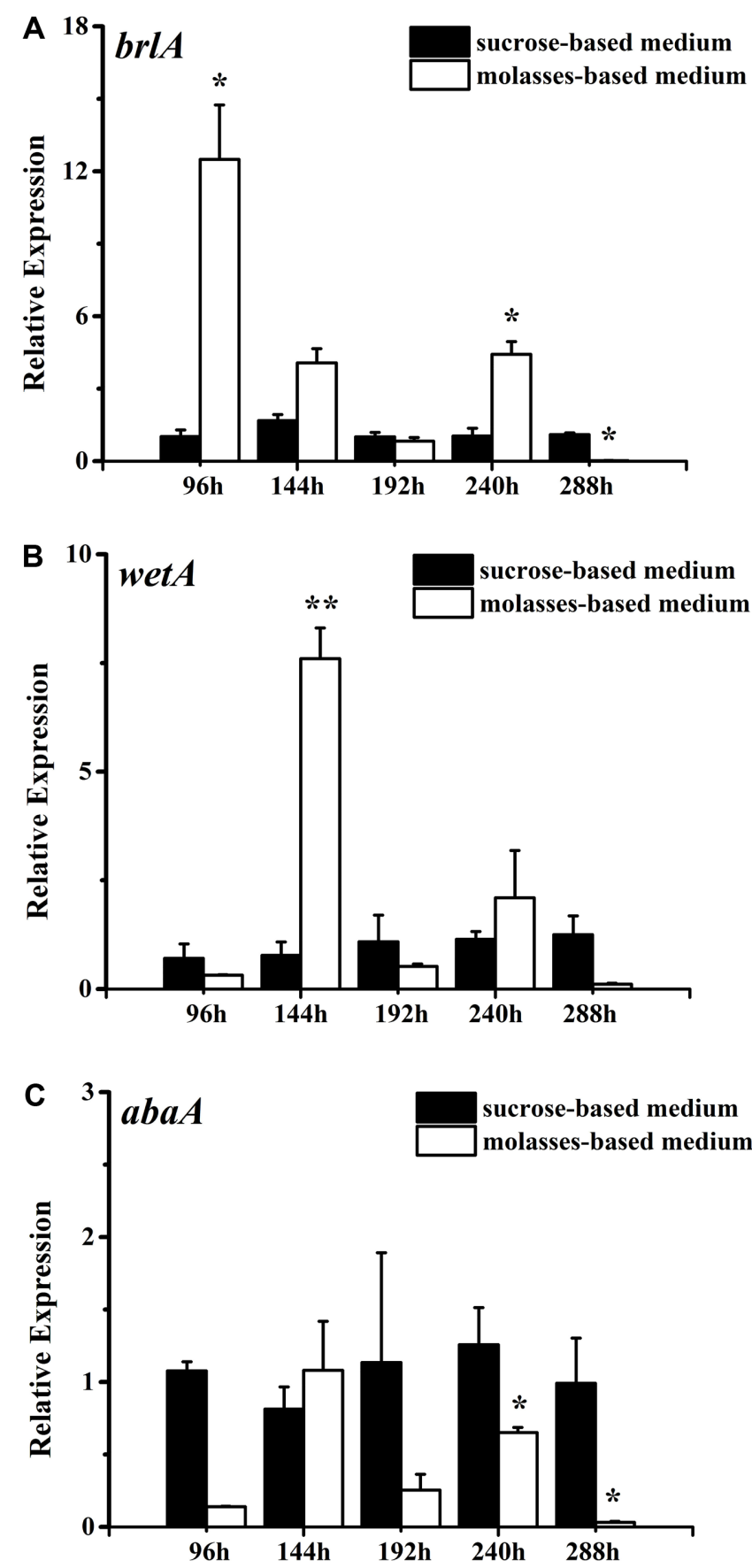

Fig. 6. Transcriptional levels of $\operatorname{brl} A(\mathbf{A})$, wet $A$ (B), and $a b a A(\mathbf{C})$. The levels of gene transcripts (B) from cultures with sucrose on day 4 were set to 1 (black column) to normalize those from cultures with molasses. The mean fold of gene transcriptional level over the control is presented for the molasses-based medium. The error bars in the figure indicate the standard deviations from three independent samples, ${ }^{*} p<0.05$.

stimulatory role of molasses, the expression of two FC biosynthetic genes, $f g a O x 1$ and $f g a O x 3$, as well as the global 

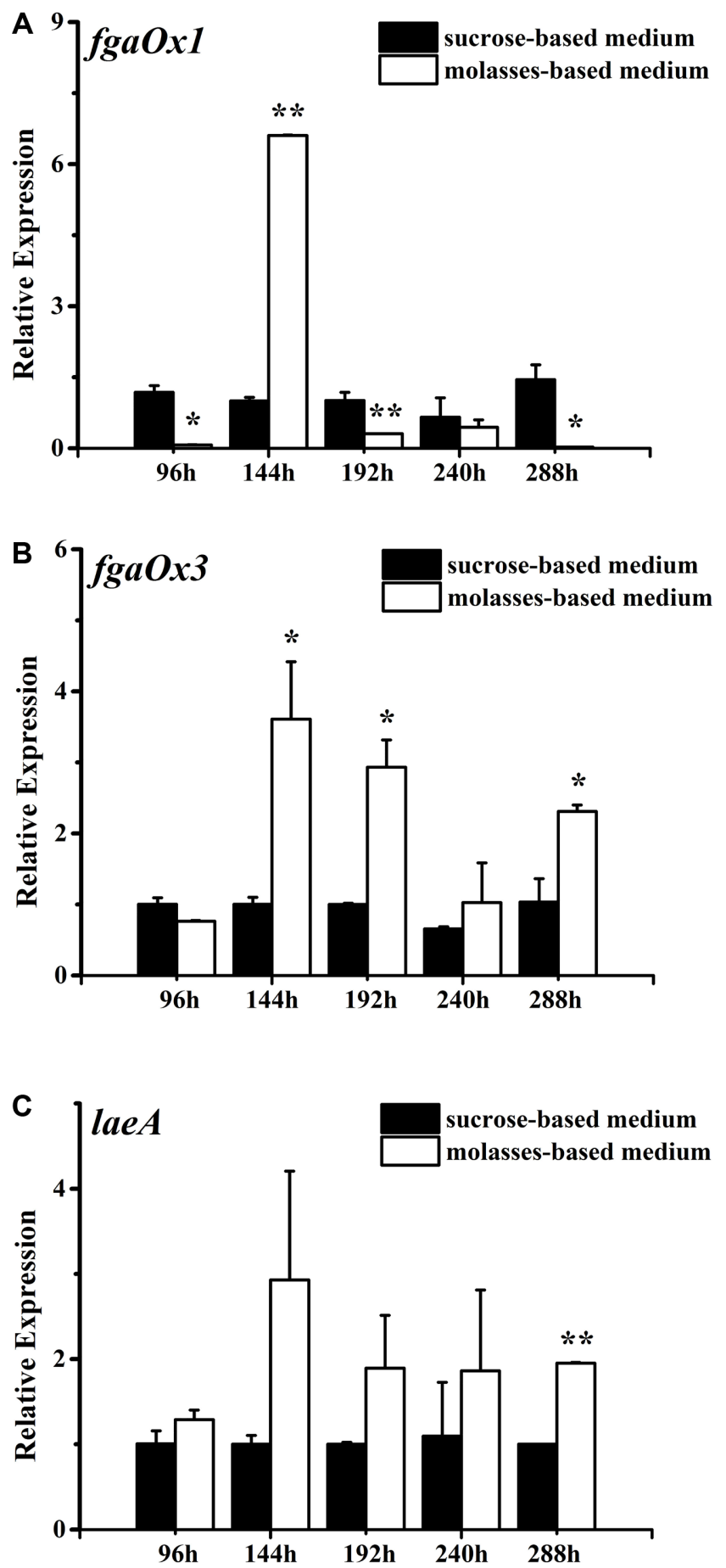

Fig. 7. Transcriptional levels of $f g a O x 1$ (A), $f g a O x 3$ (B), and laeA $(\mathbf{C})$.

The levels of gene transcripts (B) from cultures with sucrose on day 4 were set to 1 (black column) to normalize those from cultures with molasses. The mean fold of gene transcriptional level over the control is presented for the molasses-based medium. The error bars in the figure indicate the standard deviations from three independent samples; ${ }^{*} p<0.05$. secondary metabolism regulatory gene lae $A$, were quantified by qPCR (Fig. 7). The two biosynthetic genes were downregulated initially, whereas laeA expression was similar to that in the control, indicating the preliminary activation of secondary metabolism in both conditions. Afterwards, the transcriptional levels of laeA, fgaOx1, and fgaOx3 were upregulated 2.92-, 6.59-, and 3.60-fold, respectively on day 3 of static culture, in accordance with the increased FC production; however, $f g a O x 1$ upregulation lasted no more than $48 \mathrm{~h}$, after which decreased expression was observed. This may suggest that $f g a O x 1$ is not limited in the FC biosynthetic cluster. However, $f g a O x 3$ expression remained higher than in the control during the fermentation process, indicating a crucial role for FgaOx3 in molassesinduced FC biosynthesis. The expression of lae $A$ remained upregulated until the end of static culture, indicating increased secondary metabolism activity in the molassesbased medium, and partially explaining the increased FC production. A similar relationship between laeA expression and productivity was observed during the fermentation of penicillin [34] and monacolin K [35]. Moreover, LaeA is also required for conidia differentiation [36]. The lae $A$ transcriptional profile seemed to be consistent with that of brlA in the initial short period stage of static culture, not synchronously. Hence, the inner correlation of lae $A$ and brlA still requires further study. Together, the results demonstrate that molasses upregulates the expression of the secondary metabolism regulatory gene lae $A$ and the biosynthetic gene $f g a O x 3$ during static culture, and transiently upregulates $f_{g} a O x 1$.

Finally, based on our study, a genetic model for regulation of FC biosynthesis and asexual development is shown (Fig. 8). The stimulatory effects of molasses on FC biosynthesis in the biofilm at the transcriptional level was first presented. The key role of the biofilm in FC biosynthesis was verified by the transcriptional analyses of asexual development and FC biosynthetic genes. Key asexual development genes ( $\operatorname{brl} A$ and $w e t A)$, biosynthetic genes $(f g a O \times 3)$, and the global secondary metabolism regulatory gene lae $A$ were upregulated in biofilm grown with molasses, indicating the induction of both asexual development and FC biosynthesis. This study provides novel insight into the stimulatory effects of molasses on biofilm growth, and may aid further research on the regulation of $A$. fumigatus FC production.

\section{Acknowledgments}

This work is sponsored by the National Natural Science Foundation of China (81741156), the Shanghai Sailing 


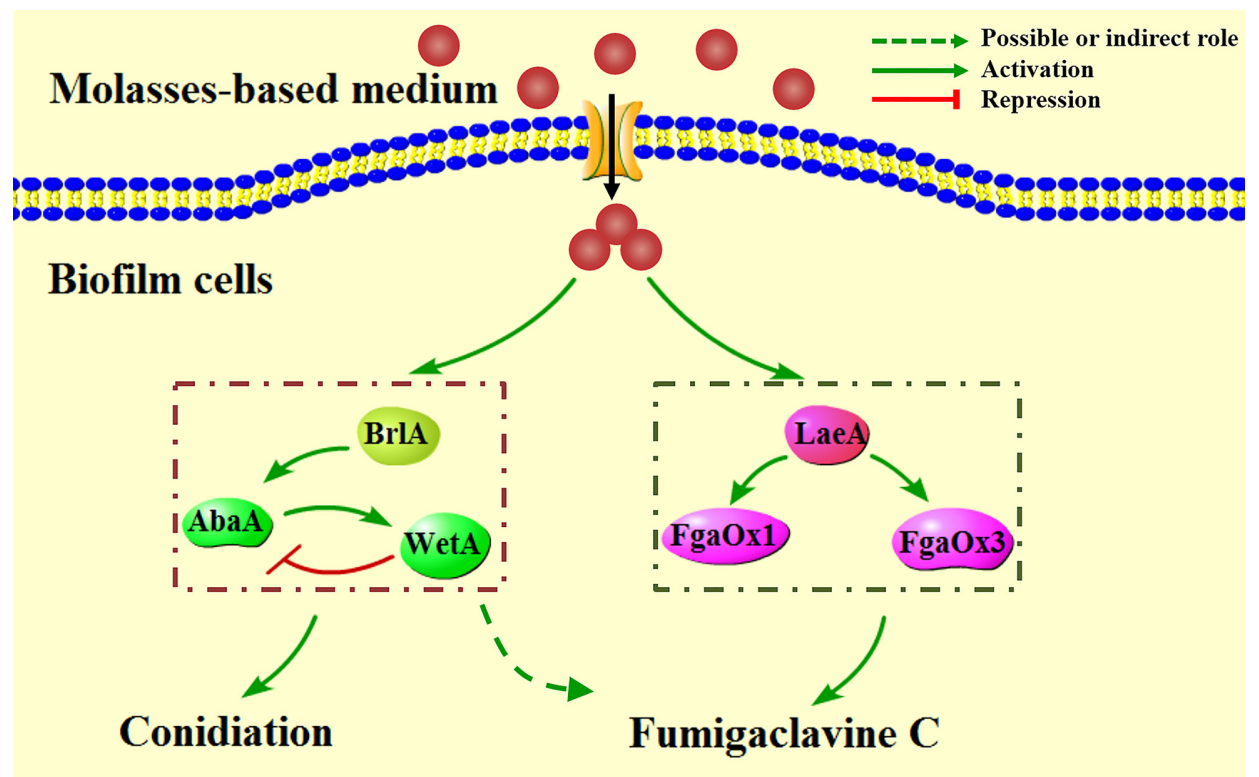

Fig. 8. Stimulatory effects of molasses on fumigaclavine $C$ biosynthesis at the transcriptional level.

Program (17YF1403700), and the Fundamental Research Funds for the Central Universities (222201714023).

\section{Conflict of Interest}

The authors have no financial conflicts of interest to declare.

\section{References}

1. Zhu YX, Yao LY, Jiao RH, Lu YH, Tan RX. 2014. Enhanced production of fumigaclavine $\mathrm{C}$ in liquid culture of Aspergillus fumigatus under a two-stage process. Bioresour. Technol. 152: $162-168$.

2. Wu XF, Fei MJ, Shu RG, Tan RX, Xu Q. 2005. Fumigaclavine C, an fungal metabolite, improves experimental colitis in mice via downregulating Th1 cytokine production and matrix metalloproteinase activity. Int. Immunopharmacol. 5: 1543-1553.

3. Zhao Y, Liu JY, Wang J, Wang L, Yin H, Tan RX, et al. 2004. Fumigaclavine $\mathrm{C}$ improves concanavalin A-induced liver injury in mice mainly via inhibiting TNF- $\alpha$ production and lymphocyte adhesion to extracellular matrices. J. Pharm. Pharmacol. 56: 775-782.

4. Li YX, Himaya SW, Dewapriya P, Zhang C, Kim SK. 2013. Fumigaclavine $\mathrm{C}$ from a marine-derived fungus Aspergillus fumigatus induces apoptosis in MCF-7 breast cancer cells. Mar. Drugs 11: 5063-5086.

5. Du RH, Qin SY, Shi LS, Zhou ZQ, Zhu XY, Liu J, et al. 2014. Fumigaclavine $\mathrm{C}$ activates PPAR $\gamma$ pathway and attenuates atherogenesis in ApoE-deficient mice. Atherosclerosis 234: 120-128.

6. Unsöld IA, Li SM. 2005. Overproduction, purification and characterization of FgaPT2, a dimethylallyltryptophan synthase from Aspergillus fumigatus. Microbiology 151: 1499-1505.

7. Wallwey C, Li SM. 2011. Ergot alkaloids: structure diversity, biosynthetic gene clusters and functional proof of biosynthetic genes. Nat. Prod. Rep. 28: 496-510.

8. Mai P, Li SM. 2013. Alkaloids derived from tryptophan: a focus on ergot alkaloids, pp. 701-702. In Ramawat KG, Mérillon JM (eds.), Natural Products. Springer, Berlin-Heidelberg.

9. Perrin RM, Fedorova ND, Bok JW, Cramer RA, Wortman JR, Kim HS, et al. 2007. Transcriptional regulation of chemical diversity in Aspergillus fumigatus by LaeA. PLoS. Pathog. 3: e50.

10. Twumasi-Boateng K, Yu Y, Chen D, Gravelat FN, Nierman WC, Sheppard DC. 2009. Transcriptional profiling identifies a role for BrlA in the response to nitrogen depletion and for StuA in the regulation of secondary metabolite clusters in Aspergillus fumigatus. Eukaryot. Cell 8: 104-115.

11. Yao LY, Zhu YX, Jiao RH, Lu YH, Tan RX. 2014. Enhanced production of fumigaclavine $\mathrm{C}$ by ultrasound stimulation in a two-stage culture of Aspergillus fumigatus CY018. Bioresour. Technol. 159: 112-117.

12. González-Ramírez AI, Ramírez-Granillo A, Medina-Canales MG, Rodríguez-Tovar AV, Martínez-Rivera MA. 2016. Analysis and description of the stages of Aspergillus fumigatus biofilm formation using scanning electron microscopy. BMC Microbiol. 16: 243 .

13. Lynch AS, Robertson GT. 2008. Bacterial and fungal biofilm infections. Annu. Rev. Med. 59: 415-428. 
14. Fanning S, Mitchell AP. 2012. Fungal biofilms. PLoS. Pathog. 8: e1002585.

15. Anderson JG, Blain JA, Divers M, Todd JR. 1980. Use of the disc fermenter to examine production of citric acid by Aspergillus niger. Biotechnol. Lett. 2: 99-104.

16. Blain JA, Anderson JG, Todd JR, Divers M. 1979. Cultivation of filamentous fungi in the disc fermenter. Biotechnol. Lett. 1: 269-274.

17. Ramage G, Rajendran R, Gutierrez-Correa M, Jones B, Williams C. 2011. Aspergillus biofilms: clinical and industrial significance. FEMS Microbiol. Lett. 324: 89-97.

18. Wang J. 2000. Production of citric acid by immobilized Aspergillus niger using a rotating biological contactor (RBC). Bioresour. Technol. 75: 245-247.

19. Ogawa A, Wakisaka Y, Tanaka T, Sakiyama T, Nakanishi K. 1995. Production of kojic acid by membrane-surface liquid culture of Aspergillus oryzae NRRL484. J. Ferment. Bioeng. 80: 41-45.

20. Aziani G, Terenzi HF, Jorge JA, Guimarães LHS. 2012. Production of fructooligosaccharides by Aspergillus phoenicis biofilm on polyethylene as inert support. Food Technol. Biotechnol. 50: 40-45.

21. Wang HL, Li P, Liu YF, Ren ZF, Wang G. 2012. Overproduction of a potential red pigment by a specific self-immobilization biomembrane-surface liquid culture of Penicillium novae-zeelandiae. Bioprocess Biosyst. Eng. 35: 1407-1416.

22. Gamarra NN, Villena GK, Gutiérrez-Correa M. 2010. Cellulase production by Aspergillus niger in biofilm, solidstate, and submerged fermentations. Appl. Microbiol. Biotechnol. 87: 545-551.

23. Bruns S, Seidler M, Albrecht D, Salvenmoser S, Remme N, Hertweck C, et al. 2010. Functional genomic profiling of Aspergillus fumigatus biofilm reveals enhanced production of the mycotoxin gliotoxin. Proteomics 10: 3097-3107.

24. Zhu YX, Hu WW, Yao LY, Yu WG, Jiao RH, Lu YH, et al. 2015. Improvement of fumigaclavine $C$ production in a twostage culture of Aspergillus fumigatus with molasses as a costeffective ingredient. Biotechnol. Bioprocess Eng. 20: 1106-1113.

25. Mowat E, Butcher J, Lang S, Williams C, Ramage G. 2007. Development of a simple model for studying the effects of antifungal agents on multicellular communities of Aspergillus fumigatus. J. Med. Microbiol. 56: 1205-1212.

26. Ali N, Yousaf S, Anam M, Bangash Z, Maleeha S. 2016. Evaluating the efficiency of a mixed culture biofilm for the treatment of black liquor and molasses in a mediator-less microbial fuel cell. Environ. Technol. 37: 2815-2822.

27. Hu X, Chen K, Lai X, Ji S, Kaiser K. 2016. Effects of Fe(III) on biofilm and its extracellular polymeric substances (EPS) in fixed bed biofilm reactors. Water Sci. Technol. 73: 2060-2066.

28. Varhimo E, Varmanen P, Fallarero A, Skogman M, Pyörälä S, Iivanainen A, et al. 2011. Alpha- and $\beta$-casein components of host milk induce biofilm formation in the mastitis bacterium Streptococcus uberis. Vet. Microbiol. 149: 381-389.

29. Yigit N, Aktas E, Dagistan S, Ayyildiz A. 2011. Investigating biofilm production, coagulase and hemolytic activity in Candida species isolated from denture stomatitis patients. Eurasian J. Med. 43: 27-32.

30. Adams TH, Wieser JK, Yu JH. 1998. Asexual sporulation in Aspergillus nidulans. Microbiol. Mol. Biol. Rev. 62: 35-54.

31. Chang PK, Hua SS. 2007. Molasses supplementation promotes conidiation but suppresses aflatoxin production by small sclerotial Aspergillus flavus. Lett. Appl. Microbiol. 44: 131-137.

32. Tudzynski P, Correia T, Keller U. 2001. Biotechnology and genetics of ergot alkaloids. Appl. Microbiol. Biotechnol. 57: 593-605.

33. Alkhayyat F, Sun CK, Yu JH. 2014. Genetic control of asexual development in Aspergillus fumigatus. Adv. Appl. Microbiol. 90: 93-107.

34. Kosalková K, Garcíaestrada C, Ullán RV, Godio RP, Feltrer R, Teijeira F, et al. 2009. The global regulator LaeA controls penicillin biosynthesis, pigmentation and sporulation, but not roquefortine $\mathrm{C}$ synthesis in Penicillium chrysogenum. Biochimie 91: 214-225.

35. Lee SS, Lee JH, Lee I. 2013. Strain improvement by overexpression of the laeA gene in Monascus pilosus for the production of monascus-fermented rice. J. Microbiol. Biotechnol. 23: 959-965.

36. Bayram ÖS, Bayram Ö, Valerius O, Park HS, Irniger S, Gerke J, et al. 2010. LaeA control of velvet family regulatory proteins for light-dependent development and fungal celltype specificity. PLoS. Genet. 6: e1001226. 